Military Technical College Kobry El-Kobbah, Cairo, Egypt

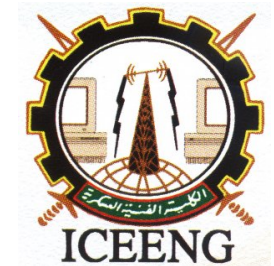

\author{
$7^{\text {th }}$ International Conference \\ on Electrical Engineering \\ ICEENG 2010
}

\title{
An Energy Efficient Routing for Wireless Sensor Networks based on a novel Cluster Head Election
}

\author{
By
}

Ashraf.Abo shosha

Ragab.Elsagher

Abd elwahab

\section{$\underline{\text { Abstract: }}$}

Wireless sensor networks (WSNs) consist of a limited capability nodes called sensors. These sensors may be in size of grain of dust in some applications so it has very limited resources (memory, energy, computational capabilities, transmission range...ect). Most of the WSN literature focuses on the efficient use of the network resources to increase the network life time as well as the system robustness. Unlike traditional networks, where the focus is on maximizing channel throughput or minimizing node deployment and enhancing the quality of service. This paper presents a new cluster head election algorithm aims to prolong the stable region of the network as well as the system life time by using the network energy efficiently. The presented technique is called the heterogeneity aware LEACH (HALEACH). It was compared to the Direct transmission (DT), the homogeneous LEACH, the heterogeneity unaware LEACH and the Fair protocol. The results show that HALEACH outperforms the other compared techniques.

* Egyptian Armed Forces

** Benha High Technology Institute, Benha, Egypt

*** College of Engineering, Cairo University, Cairo, Egypt 


\section{Introduction:}

Classical control theory proves to be insufficient in modeling distributed control problems where issues of communication delay, jitter, and time synchronization between components are not negligible. So the distributed control system (DCS) was introduced to be the alternative. The distributed control systems use the sensors as key elements. Some problems arise when we use the wired sensor networks in control applications especially when the applications require that we change the sensor position or put it in an ad-hoc manner. So the distributed control system within the wireless adhoc sensor network became the new trend because this technology enables us to easy install our control network even by non technical persons. Also it's cost effective, Self configurable, easy to deploy, secure and undetectable. So the WSN are recently emerged in many applications and industries which include but not limited to the military applications, telemedicine, modern agriculture and oil and gas industries. The wireless sensor networks are used in the military applications to sense the battle field and the enemy movements and sending this data to the base station to take the decision accordingly. Also it can be used in telemedicine to monitor the patient behavior to the specialist. The wireless sensor networks can also be used in modern agriculture to measure the environmental variables. It used also in intelligent transportation systems and home automation and other industries. WSN are used in oil and gas industries to collect the data of the well heads and the remote area facilities and send them to the distributed control system (DCS) in order to take the proper action. These collected data contain an analog signals like the well head flow line pressure, temperature and the choke valve position and also digital signals like if there's fire or no and if there's local emergency shutdown ( local ESD) or no. For example if the flow line pressure high the DCS proper action will be to reduce the choke valve opening in order to protect other well heads from backpressure. Due to the variety of its applications wireless ad-hoc sensor networks have recently emerged as a premier research topic. They have great long-term economic potential, and pose many new system-building challenges. Sensor networks are gaining a central role in the research community. This framework focuses on prolonging the life time of the network and increasing the system reliability by using the network energy in an efficient manner.

The paper is organized as follows; Section 2 gives a brief overview of the previous related work. In this section, we survey the state-of-the-art routing protocols for WSN. Section 3 formulates the problem that we aim to study. In section 4 we state some important definitions as well as the performance metrics that will be used to evaluate the proposed schemes. The simulation model characteristics were mentioned in section 5 . Section 6 gives an overview of the low energy adaptive clustering hierarchy (LEACH) protocol proposed by Heinzelman to consume the network energy and prolong the 
network life time. An enhancement to the LEACH was presented in section 7. Section 8 states an analytical study for the WSN energy model. The obtained results and the result analysis were discussed in section 9. Section 10 states the conclusion and the future work.

\section{Survey of the related work:}

In computer science and telecommunications, wireless sensor networks are an active research area with numerous workshops and conferences arranged each year due to its importance and the variety of its applications. Sensor networks also pose a number of new conceptual and optimization problems that may be a hot area. Sensor networks pose the number of unique technical challenges due to some factors like the ad hoc deployment since most sensor nodes are deployed in regions which have no infrastructure at all. A typical way of deployment in a forest would be tossing the sensor nodes from an airplane. In such a situation, it is up to the nodes to identify its connectivity and distribution. Also the unattended operation in most cases, once deployed, sensor networks have no human intervention. Hence the nodes themselves are responsible for reconfiguration in case of any changes. Also one of these factors is the dynamic changes since It is required that a sensor network system be adaptable to changing connectivity (for e.g., due to addition of more nodes, failure of nodes etc.) as well as changing environmental stimuli. And finally, the untethered where the sensor nodes are not connected to any energy source. There is only a finite source of energy, which must be optimally used for processing and communication. An interesting fact is that communication dominates processing in energy consumption. Thus, in order to make optimal use of energy communication should be minimized as much as possible. Thus, unlike traditional networks, where the focus is on maximizing channel throughput or minimizing node deployment, the major consideration in a sensor network is to extend the system lifetime as well as the system robustness [7].

A number of papers propose solutions to one or more of the above problems. Our survey focuses on the suggested solutions in Energy Efficiency. Energy efficiency is a dominant consideration no matter what the problem is. This is because sensor nodes only have a small and finite source of energy. Many solutions, both hardware and software related, have been proposed to optimize energy usage. An extensible ondemand power management framework for ad hoc networks that adapts to traffic load was proposed in [1]. We may use the location information in energy efficient routing of WSN as the SLEAR protocol [2]. A cross-Layer Scheduling for Power Efficiency in Wireless Sensor Networks was presented in [3]. The approach will not be suitable for event driven sensor networks, where the schedule setup overhead will likely outweigh the power savings. The approach does not fit cleanly in any one layer, as it require the collaboration of both the routing and MAC layers. [4] studied the application of the Ant 
Colony Optimization metaheuristic to solve the routing problem in wireless sensor networks. The resulting routing protocol, called Energy- Efficient Ant Based Routing (EEABR), uses lightweight ants to find routing paths between the sensor nodes and the sink nodes, which are optimized in terms of distance and energy levels. An online heuristic was proposed in [5] to maximize network lifetime. This heuristic performs two shortest path computations to route each message. Jyh-Huei Chang and Rong-Hong Jan (2006) [6] presented an energy aware cluster based routing algorithm for wireless sensor networks. They compared their solution (ECRA scheme) and proved that it can achieve longer lifetime. This is because the ECRA rotates intra-cluster-heads to balance the load to all nodes in the sensor networks. The numerical results showed that ECRA gives better performance in load-balancing and energy-saving than other schemes. Levente Butty and Peter Schaffer (2007) [7] introduced PANEL, a position-based aggregator node election protocol for wireless sensor networks. The novelty of PANEL with respect to other aggregator node election protocols is that it supports asynchronous sensor network applications where the sensor readings are fetched by the base stations after some delay. A distributed, randomized clustering algorithm to organize the sensors in a wireless sensor network into clusters was proposed in [8]. This algorithm was extended to generate a hierarchy of cluster heads and observe that the energy saving increase with the number of levels in the hierarchy. Results in stochastic geometry are used to derive solutions for the values of parameters of their algorithm that minimize the total energy spent in the network when all sensors report data through the cluster heads to the processing center. The drawback of this previous work is that they only focus on prolonging the network life time only. In this framework we aim to prolong the stable region of the network as well as the life time where some applications work only in the stable region

\section{Problem formulation :}

In WSN there are two alternative routing approaches; flat multi-hop routing and clustering based routing. In flat multi-hop routing we either use minimum transfer energy "MTE" or direct transmission "DT". Every one of them has its drawbacks. In MTE every node will forward its reports to the neighbor node until the report reaches the base station so the base station neighbor nodes will be used as relay many times and will die fast hence the stable region of the network will be short. In DT, every node will send directly to the sink so the far nodes will have a long transmission distance to reach the sink and hence will die fast. The stable region of the network will be short also. Due to this drawback, the optimum solution will be the clustering techniques. It may be advantageous to organize the sensors into clusters to communicate the data over smaller distances which will costs a low transmission distance. One of the most famous clustering techniques is the low energy adaptive clustering technique(LEACH) [9], [10]. 
In LEACH every subset of nodes will send its reports to one of them which called the cluster head. The cluster head will relay these reports to the sink. The cluster head role will be rotated between all nodes in the cluster and also the clusters are reestablished to distribute the dissipated energy. A detailed description of LEACH will be in section 6 . There's many methods to improve the performance of LEACH by increasing the hierarchical clustering levels, By the use of combined LEACH and MTE routing with the cluster heads acting as routers, to ensure that the cluster head nodes are uniformly distributed by dividing advertisement phase into smaller sub-phases or by using the energy based threshold function. This framework aims to enhance the performance of LEACH by proposing a cluster head election technique which prolongs the stable region of the network by reducing the total energy dissipation.

\section{The performance metrics:}

To state our performance metrics we should mention some definitions:

The round: the process of sending one report from every node to the base station directly (in direct transmission) or via the cluster head in clustering.

The epoch: no of rounds that enable every node in the cluster to have the chance to be cluster head at least one time.

The stable region: the time between the start of operation of the network and the first node death (FND). Also we denote the half node death denoted as MND.

The unstable region: the time between the first node death and the last node death (LND).

The network life time: the time from the start of operation till the death of the last node so it equal summation of the stable and unstable region. In this article we are going to take number of alive nodes per round, the first node death (FND), MND and the last node death (LND) as a metrics to evaluate our proposed technique in compare to the other techniques. Cluster-based routing protocol has special advantages to enhance scalability and efficiency of the routing protocol.

\section{The simulation model:}

We will construct a WSN which consist of 100 nodes uniformly distributed over $100 \mathrm{~m}$ $* 100$ m distribution region figure 1 . These nodes are stationary not mobile. The sink is in the middle of the network and assumed to have sufficient resources at least compared to the sensor nodes. The message size equals 4000 bit and the initial energy for normal node is 0.5 joules. The data aggregation energy cost $E_{D A}$ is 5 Nan joule. The transmit energy and receive energy $E_{T X}$ and $E_{R X}$ is 50 Nan joule. The transmit amplifier has $\varepsilon_{\mathrm{mp}}$ and $\varepsilon_{\text {fs }}$ equals 0.0013 and 10 pjoule $/ \mathrm{bit} / \mathrm{m}^{\wedge} 2$. 


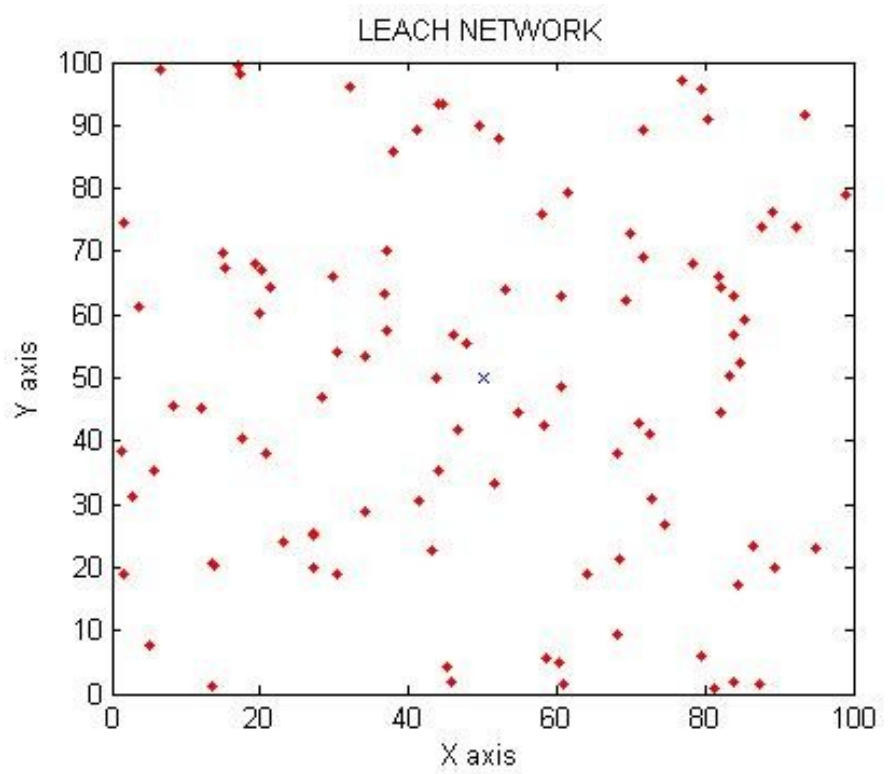

Figure (1): a typical WSN model

\section{The low energy adaptive clustering hierarchy (LEACH):}

The low energy adaptive clustering hierarchy protocol (LEACH) proposed in [9] divides the network into clusters where every cluster has its own cluster head which receive the reports from the cluster nodes and perform data aggregation in order to reduce the amount of data that will be transmitted to the base station. And then it utilizes randomized rotation of local cluster heads to evenly distribute the energy load among the sensors in the network. In LEACH, the sensor nodes may be homogeneous in its initial energy which we call it (homogeneous LEACH) but the most practical situation for these nodes to be heterogeneous (heterogeneous LEACH) since this heterogeneity result in the network operation. In the wireless sensor network community, the LEACH protocol has many advantages where it reduces the energy dissipation compared to direct communication DC and compared to MTE routing. Where in DT, the nearest nodes to the sink dies first and vise versa for MTE but in LEACH nodes die randomly which increase the reliability of the network. LEACH requires no global knowledge of the network. Although LEACH uses dynamic clustering which may bring extra overhead it increases the system lifetime. The operation of LEACH is broken up into rounds, where each round begins with a set-up phase followed by a steady-state phase; see the flow chart (figure 2).

\subsection{The set up phase includes):}

6.1.1 Cluster-head Advertisement

Initially each node decides whether or not to become a cluster-head for the current 
round. This decision is made by the node $\mathrm{n}$ choosing a random number between 0 and 1 . If the number is less than a threshold $\mathrm{T}(\mathrm{n})$, the node becomes a cluster-head for the current round. The threshold is set as:

$T(n)=\frac{p}{1-p\left(r \operatorname{Mod} \frac{1}{p}\right)}$

0 if $\mathrm{n} \in G$.

otherwise

Where $\mathrm{P}=$ the desired percentage of cluster heads, $\mathrm{r}=$ the current round, and $\mathrm{G}$ is the set of nodes that have not been cluster-heads in the last 1/P rounds. There exist an optimal number of nodes that should be cluster heads which is $k_{p o p t}=n p_{p o t}=2.62 \sqrt{\frac{n}{2 \pi}}$ as we will deduce later in section 8. Each node that has elected itself a cluster-head for the current round broadcasts an advertisement message to the rest of the nodes. For this "clusterhead-advertisement" phase, the cluster-heads use a carrier sense multiple access-media access control (CSMA MAC) protocol, and all cluster-heads transmit their advertisement using the same transmit energy. After this phase is complete, each noncluster-head node decides the cluster which it will belong for this round. This decision is based the received signal strength of the advertisement. In the case of ties, a random cluster-head is chosen.

Cluster Set-Up

After each node has decided to which cluster it belongs, it must inform the cluster-head node that it will be a member of the cluster. Each node transmits this information back to the cluster-head again using a CSMA MAC protocol.

6.1.3 Transmission schedule creation

The cluster-head node receives all the messages for nodes that would like to be included in the cluster. Based on the number of nodes in the cluster, the cluster-head node creates a time division multiple access (TDMA) schedule telling each node when it can transmit. This schedule is broadcast back to the nodes in the cluster. Transmission in one cluster will affect (and hence degrade) communication in a nearby cluster. To reduce this type of interference, each cluster communicates using different code division multiple access (CDMA) codes. Efficient channel assignment is a difficult problem.

\subsection{The Steady-state phase includes):}

\subsubsection{Data transmission to cluster heads}

Once the clusters are created and the TDMA schedule is fixed, data transmission can begin. Nodes send their data during their allocated transmission time to the cluster head. This transmission uses a minimal amount of energy (chosen based on the received strength of the cluster-head advertisement). The radio of each non-cluster-head node can 
be turned off until the node's allocated transmission time, thus minimizing energy dissipation in these nodes.

\subsubsection{Signal processing (Data fusion)}

When all the data has been received, the cluster head node perform signal processing functions to compress the data into a single signal.

6.2.3 Data transmission to the base station

The cluster head sends this composite signal to the base station. 


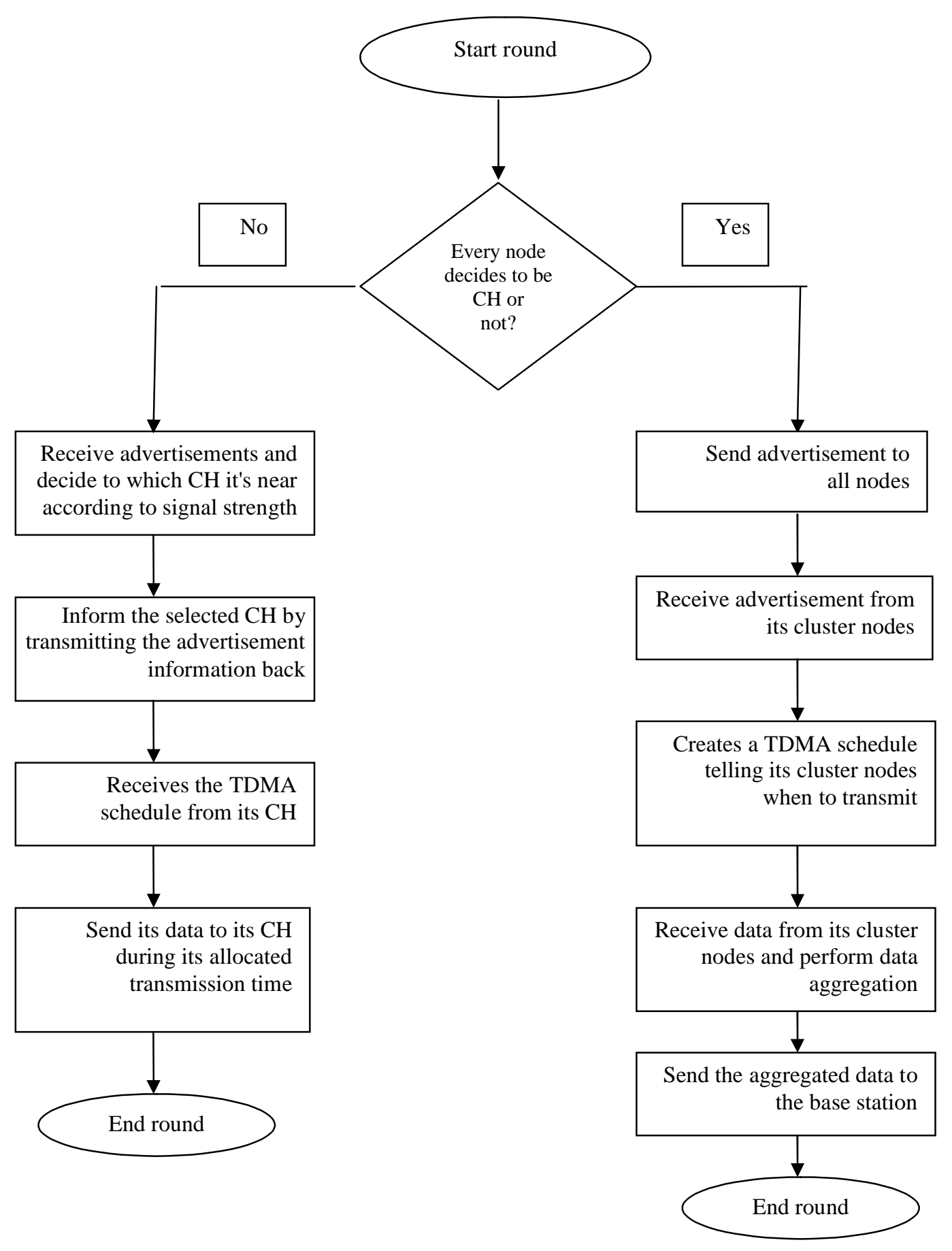

Figure (2): Flow chart of LEACH protocol 


\section{The proposed technique:}

LEACH is promising routing protocol for the sensor networks, however there were not enough experimental data on different network topologies and different radio parameters to make any final conclusions. Further study is required to estimate the full potential of the LEACH routing protocol. Most of the researchers in LEACH protocol obtained their results assuming that the sensor nodes are equipped with the same amount of energy as proposed in [9] and [3]. In this article we call that the homogeneous LEACH. But this situation isn't realistic even if it was at the initialization of the network, it wouldn't be realistic later where every node consumes the energy with different rate than the other nodes due to its transmission distance so the heterogeneity of the nodes in terms of their energy is simply a result of the network operation. If the transmission distance of the node increases the energy dissipation in this node will increase which will cause the heterogeneity of the network later. Also there are a lot of applications that would benefit from such heterogeneity. One of these applications could be the reenergization of the network nodes. Here we will use the heterogeneous model of the network which is the most practical model. We will benefit this heterogeneity to propose a new cluster head rotation mechanism (heterogeneous aware LEACH) that will save the network energy and hence increase the network life time. In the heterogeneous aware LEACH the network will equipped with some nodes that have extra energy than the other nodes. These nodes are called the alpha nodes and will have more probability to be a cluster head than the other nodes. The fraction of the alpha nodes $\mathrm{m}$ where $m$ no of alpha nodes

, where $\mathrm{n}$ the total number of sensor nodes. These alpha nodes are equipped with $a$ extra energy. The regular node will have an initial energy $E_{0}$ and the alpha node will have an initial energy $E_{0}(1+a)$. So the total (initial) energy of the new heterogeneous setting will equal the summation of the initial energy of both the alpha nodes and the regular nodes and will be given by the equation:

The Total initial energy $=n(1-m) E_{0}+n m E_{0}(1+a)=n E_{0}(1+a m)$

Let's define $p$ the probability that a node be a cluster head. So the epoch will equal $\frac{1}{p}$ in the homogeneous LEACH. But here in our model the initial energy increased by factor $(1+a m)$ as (2). So the epoch will be increased by the same factor to be $\frac{1}{p}(1+a m)$ and this is the first improvement. We can increase the stable region of the network 
by $(1+a m)$ times only if each regular node becomes a cluster head once every $\frac{1}{p}(1+a m)$ round per epoch and every alpha node become a cluster head $1+a$ times. This means that every node has to become a cluster head as many times as its initial energy divided by the energy of the regular node. But the average number of cluster heads per round must still equal to ${ }^{(n p)}$ as the previous LEACH model since we didn't change the spatial density. Increasing the initial energy for the alpha nodes is equivalent to adding (nam) virtual nodes. So in the presented scheme the total number of nodes including real and virtual nodes will be $n(1+a m)$. The weighted probabilities for regular nodes is $p_{\text {reg }}=\frac{p}{1+a m}$ and for the alpha nodes $\quad p_{\alpha}=\frac{p}{1+a m}(1+a)$ and the average number of cluster heads per round per epoch is equal to $n(1+a m) p_{\text {reg }}$, to obtain the threshold that is used to elect the cluster head in each round we replace $\mathrm{p}$ in equation (1) by $p_{\text {reg }}$ or $\mathrm{p}_{\alpha}$ :

$\mathrm{T}\left(\mathrm{n}_{\text {reg }}\right)=\frac{p_{\text {reg }}}{1-p_{\text {reg }}\left(r \operatorname{Mod} \frac{1}{p_{\text {reg }}}\right)}$

0

$\mathrm{T}\left(n_{\alpha}\right)=\frac{p_{\alpha}}{1-p_{\alpha}\left(r \operatorname{Mod} \frac{1}{p_{\alpha}}\right)}$

0 if $\mathrm{n}_{\mathrm{reg}} \in G_{1}$.

otherwise

if $\mathrm{n}_{\alpha} \in G 2$.

otherwise

Where $\mathrm{T}(\mathrm{n}$ reg $)$ the threshold applied to a population of $n \cdot(1-m)$ regular nodes, G1 is the set of regular nodes that have not become cluster heads within the last 1

$\mathrm{p}$ reg round of the epoch. And $T\left(n_{\alpha}\right)$ is the threshold applied to a population of $n . m$ alpha nodes, $\mathrm{G} 2_{2}$ is the set of advanced nodes that have not become cluster heads within the last $\frac{1}{p_{\alpha}}$ rounds of the epoch. This guarantees that each regular node will become a cluster head exactly once every $\frac{1}{p} \cdot(1+a \cdot m)$ rounds per epoch and every alpha node will become a cluster head once exactly every $\frac{1}{p} \cdot \frac{1+a \cdot m}{1+a}$ rounds 
per epoch and this period defined as sub-epoch. The heterogeneous epoch equals $(1+a)$ sub-epoch and so each alpha node will become a cluster head exactly $(1+a)$ times within the heterogeneous epoch. The average number of cluster heads per round per heterogeneous epoch equals to:

$$
n .(1-m) p_{\text {reg }}+n . m p_{\alpha}=n \cdot p_{\text {opt }}
$$

Where $p_{o p t}=\frac{k_{o p t}}{n}$ and this value of ${ }^{k_{o p t}}$ is the optimum value of clusters number which yields minimum value for the total energy dissipation $E_{\text {tot }}$.

\section{The energy model}

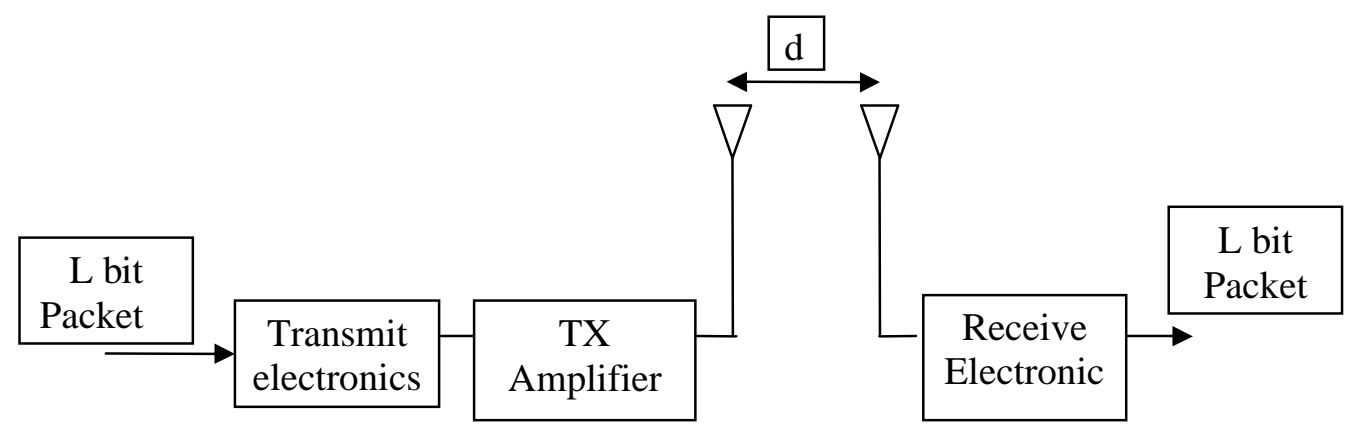

Figure (3): the first order radio dissipation model

In figure 3 to transmit $\mathrm{L}$ bit over distance $\mathrm{d}$ with acceptable signal to noise ratio SNR, the energy dissipated by the transmitter is given by the following equation:
$E_{T X}(1 . d)=L E_{\text {elec }}+L \cdot \varepsilon_{f s} d^{2}$
if $\mathrm{d} \leq \mathrm{do}$
$=L E_{\text {elec }}+L \varepsilon_{m p} d^{\wedge} 4$
if $\mathrm{d} \succ d o$

Where $\mathcal{E}_{\mathrm{fs}}, \mathcal{E}_{\mathrm{mp}}$ depend on the amplifier model, Eelec is the energy dissipated by the transmitter electronic circuit and $\mathrm{d}$ the distance between the sender and the receiver. By equating equation (5) and (6) at $d=d_{o}$, we will get: 
$d_{0}=\sqrt{\frac{\varepsilon_{f s}}{\varepsilon_{m p}}}$

Also to receive $\mathrm{L}$ bit message the radio expends:

$E_{R X}=L E_{\text {elec }}$

Assume the distance of any node to the sink or to its cluster head to be $\leq \mathrm{d}_{\mathrm{o}}$ for simplicity.

$$
\begin{aligned}
& E_{C H}=E_{R X}+E_{D A}+E_{T X} \\
& E_{C H}=\left(\frac{n}{K}-1\right) L E_{\text {elec }}+\frac{n}{k} L E_{D A}+L E_{\text {elec }}+L \varepsilon_{f s} d^{2}{ }_{\text {toBS }} \\
& E_{\text {nonCH }}=E_{T X}=\left(L E_{\text {elec }}+L \varepsilon_{f s} d_{\text {toCH }}^{2}\right) \\
& E_{\text {tot }} \approx K E_{C H}+n E_{\text {nonCH }} \\
& E_{\text {tot }}=L 2 n E_{\text {elec }}+n L E_{D A}+\varepsilon_{f s} L\left(K d_{\text {toBS }}^{2}+n d_{t o C H}^{2}\right)
\end{aligned}
$$

If we differentiate the last equation with respect to $\mathrm{k}$ and equating to zero the optimal number of clusters to minimize $E_{\text {tot }}$ can be found:

$K_{\text {opt }}=2.62 \sqrt{\frac{n}{2 \pi}}$

Where $d_{t o B S}=0.3825 M, d_{t o C H}=\sqrt{\frac{M^{2}}{2 \pi K}}$, where the field area $\mathrm{A}=\mathrm{M} \times \mathrm{M}$ square meters. The same analysis can be done for $\mathrm{d} \succ \mathrm{d}_{\mathrm{o}}$.

\section{The simulation result and analysis:}

Most of the energy-efficient protocols depend on the network topology and radio parameters of the system. By using matlab simulator here we simulated the direct 
transmission protocol (DT). The result in figure 4 shows that all nodes will die after approximately 20 round which is a very short life time. Figure 5 shows the number of alive nodes for various LEACH techniques, homogeneous LEACH, heterogeneity unaware LEACH and the proposed heterogeneity aware LEACH protocol. Also the results if we redistributed the extra energy to all nodes in a fair manner which called Fair are shown in the same figure. We conclude that the stable region for the heterogeneity aware LEACH is larger than the other techniques. It's near to that of Fair whereas the unstable region and hence the total system life time is larger than Fair. Usually we make a trade off between the life time and the reliability of the system. The reliability increases if the unstable region decreases and vise versa for the system life time. Some applications need all nodes to be alive so in this case the reliability will be the most important issue. Other applications need even one node to be alive for long period so in this case the life time will be the most important issue. Our approach yields both reliability and life time. The total energy dissipation is illustrated in figure 6 . The maximum total energy dissipation in the homogeneous LEACH is less than the other schemes since no extra energy was added. The stable region for the heterogeneity aware LEACH is larger than the other approaches. It's near to that of Fair but the life time for the heterogeneity aware is larger than Fair. Figure 7 shows the first, half and last node death (FND, MND and LND). The life time in the heterogeneity unaware is 1.25 times larger than that of the heterogeneity aware but the heterogeneity aware has a stable region 1.30 times larger than it. The system life time also is 2.15 times larger than Fair.

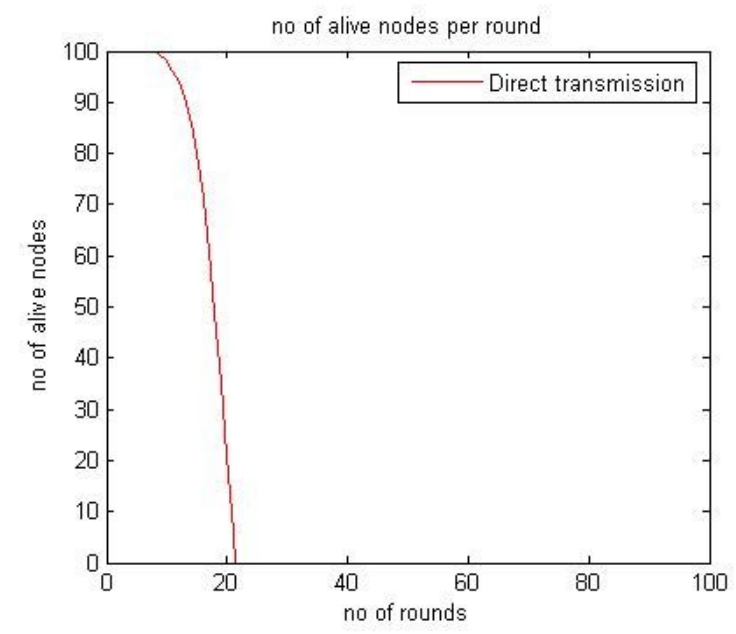

Figure (4): no of alive nodes per round for DT 


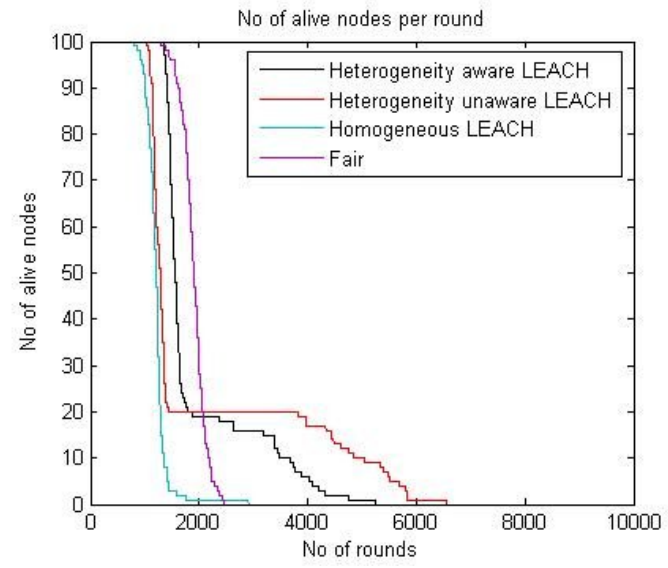

Figure (5): No of alive nodes per round comparison

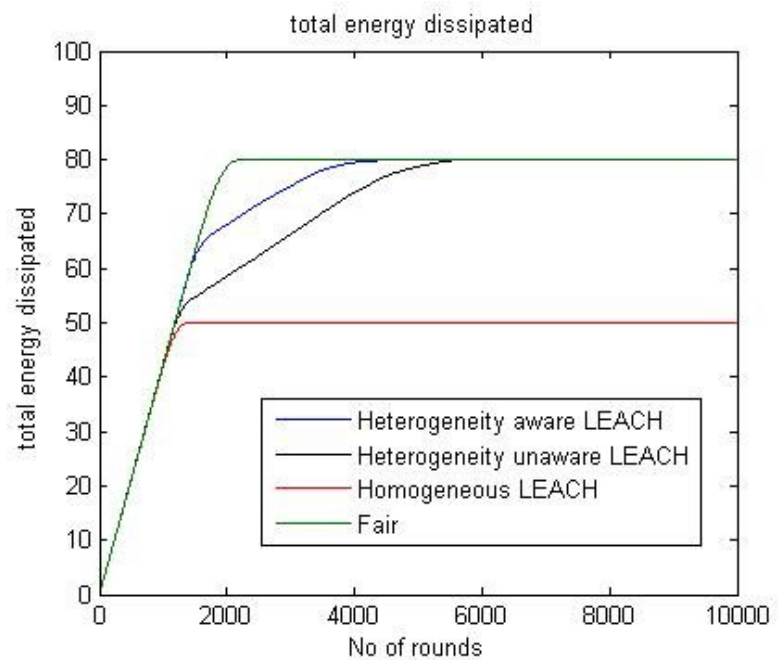

Figure (6): total energy dissipated per round comparison 


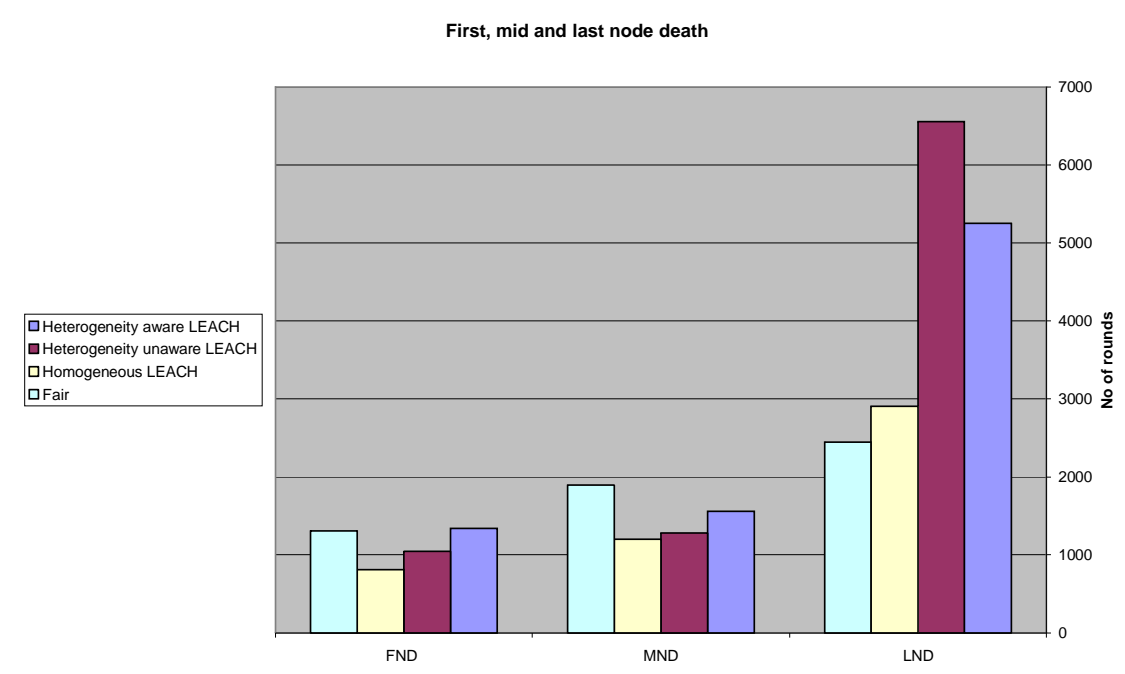

Figure (7): $F N D, M N D$ and LND comparison

\section{Conclusion and future work:}

We benefit from the heterogeneity of the network to propose a new cluster head rotation mechanism that will save the network energy and hence increase the network life time and prolong the stable region to enhance reliability of the network. The results shows that the heterogeneity aware LEACH outperforms the homogeneous LEACH, heterogeneity unaware LEACH and FAIR in terms of the proposed metrics. As a future work we will study the impact of using three level heterogeneity in the network life time. Also we will study this proposed technique in three level hierarchies WSN.

\section{References:}

[1] Rong Zheng, Robin Kravets "On-demand Power Management for Ad Hoc Networks," 0-7803-7753-2/03 IEEE, INFOCOM 2003.

[2] George Lukachan and Miguel A. Labrador "SELAR: Scalable Energy Efficient Location Aided Routing Protocol for Wireless Sensor Networks," in Proceedings of the 29th Annual IEEE International Conference on Local Computer Networks.

[3] Mihail L. Sichitiu "Cross-Layer Scheduling for Power Efficiency in Wireless Sensor Networks," 0-7803-8356-7/04 in IEEE INFOCOM 2004.

[4] Tiago Camilo, Carlos Carreto, Jorge Sá Silva1and Fernando Boavida1 "An Energy-Efficient Ant-Based Routing Algorithm for Wireless Sensor Networks" in IEEE,INFOCOM 2005. 
[5] Joongseok Park Sartaj Sahni "Maximum Lifetime Routing in Wireless Sensor Networks" National Science Foundation under grant ITR-0326155 in June, 2005.

[6] Jyh-Huei Chang and Rong-Hong Jan "An Energy Aware, Cluster-Based Routing Algorithm for Wireless Sensor Networks" in IFIP International Federation for Information Processing 2005, 44.

[7] Levente Butty and Peter Schaffer " PANEL: Position-based Aggregator Node Election in Wireless Sensor Networks" supported by the Hun-garian Scientific Research Fund and the HSN Lab. 1-4244-1455-5/07, 2007 IEEE. 